\title{
User centric media research challenges
}

\author{
Cristina Emilia Costa
}

Published online: 16 April 2014

(C) Springer Science+Business Media New York 2014

Now more than ever users are central to media: as creators, as consumers, as learners, etc. "User Centric Media" is an field that has been constantly increasing, and involves various areas of research. It implies taking into account the intrinsic user-centered nature of the media, at different levels of different media value chains. This perspective puts the user at the center during media creation, discovery, distribution and consumption, and in their applications to entertainment, education, information and arts. The papers in this special issue have the aim to help to understand, to predict and to enable trends in User Centric Media in the context of Future Media Internet. This issue consist of six papers, which are briefly discussed as follows.

When talking about media delivery and consumption, an important role is played by mobile devices. Current mobility protocols and architectures are mainly targeted to devices or applications, and usually lack the ability to support user-centric paradigms. In "User-centric mobility management for multimedia content access", the authors Raffaele Bolla, Riccardo Rapuzzi and Matteo Repetto propose a generic mobility framework for terminal handover and session migration that pursues the user-centric paradigm leveraging on a cross-layer architecture, thus yielding to a high level of generality, applicability and flexibility.

The paper "An architectural approach towards Future Media Internet" by Theodore Zahariadis, Federico Alvarez, and John Paul Moore Olmstead focus on the requirements coming from emerging immersive and real-time applications and services, and on the main architectural functions needed to support them. To overcome the current content delivery limitations, the authors present a new protocol stack and a holistic network architectural approach that targets Future Media Internet (FMI), thus proposing solutions that aim at moving intelligence in the network and converting it into a content oriented/centric network (going a step further the current CDNs) and supporting functionalities for producing, publishing, caching, finding and consuming content.

Variable network conditions entail variable quality levels, therefore nowadays applications implement some kind of dynamic service adaptation in order to mitigate these effects. Being able to identify the main sources of degradation at the destination endpoint improves the adaptation capabilities and enhances the service performance in terms of perceived quality. In the paper entitled "QoE-driven and network-aware adaptation capabilities in mobile multimedia applications" by Jose Oscar Fajardo, Ianire Taboada and Fidel Liberal, an analysis of mobile multimedia services, with special focus on current media resolutions for mobile handsets, is presented. The authors analyse the service performance from an end-to-end

C. E. Costa $(\bowtie)$

Well Group, CREATE-NET Research Center, Trento, Italy

e-mail: cristina.costa@create-net.org 
perspective, and, taking into account the several agents involved in the service provision, they discuss the different possible sources of degradations and their impact into the expected quality as perceived by end users.

For enjoying the content to its full extent, it is imperative that its access and consumption is user centric: therefore during content delivery a particular attention must be played to the quality perceived by the use, especially when considering new media types of content such as immersive interactive environments or $3 \mathrm{D}$ video. In "Modeling user perception of $3 \mathrm{D}$ video based on ambient illumination context for enhanced user centric media access and consumption", the authors Gokce Nur, Hemantha Kodikara Arachchi, Safak Dogan and Ahmet M. Kondoz discuss the important factors that influences the 3D video perception, the modeling of video quality and depth perception of $3 \mathrm{D}$ video. Based on this study the authors propose new video quality and depth perception models that can be used to assist efficient provision of user centric media.

In the paper "Automatic generation of 3D outdoor and indoor building scenes from a single image", the authors Georgios Vouzounaras, Petros Daras and Michael G. Strintzis present a novel approach for creating 3D models of building scenes, that allows, using only one image, to accurately reconstruct in real-time both outdoor images of a building and indoor scenes, with perspective cues. The proposed method is fully automated and fast. It combines the extracted line segments to identify the vanishing points of the image, the orientation, the different planes that are depicted in the image and concludes whether the image depicts indoor or outdoor scenes. In addition, the proposed method efficiently eliminates the perspective distortion and produces an accurate 3D model of the scene without any intervention from the user.

Challenges still remain in video streaming in many scenarios. Limited home broadband and mobile phone $3 \mathrm{G}$ bandwidths mean many users stream videos at compromised quality. To provide additional bandwidth for streaming Thangam Vedagiri Seenivasan and Mark Claypool in their paper "CStream: neighborhood bandwidth aggregation for better video streaming" propose a system that aggregates bandwidth from multiple cooperating users into a neighborhood environment for better video streaming that allows a linear increase in throughput over single-connection streaming and an improved video quality as the number of cooperating users in a neighborhood increases.

The six papers cover various aspects related to the User Centric Media research domain, approaching the topic from different perspectives.

We thank all authors for their contributions to this special issue. In particular, the editors would like to express their gratitude to the reviewers for their hard work. Their feedback and rigorous observations and comments significantly helped to improve the initial submissions. Without them, this work would not have been possible. 


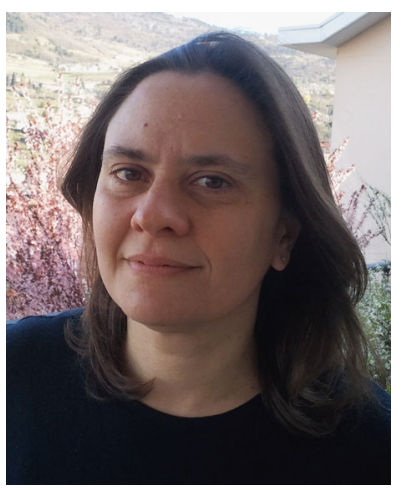

Cristina Emilia Costa is senior researcher in the WELL group at CREATE-NET. She obtained her Master degree in Electronics and Communications Engineering from the University of Genoa, Italy, in 1996. From 1997 to 2001 she worked as a researcher in TILAB, where she developed experience in the fields of Internet Services and Security. Later, she received the $\mathrm{PhD}$ degree from the International Graduate School of Information and Communication Technologies, University of Trento, with a thesis on image and video compression and transmission over wireless networks. Dr. Costa research focus is on is on the development and evaluation of innovative solutions for the acquisition and maintenance of healthy lifestyles. 